\title{
All-optical phase regeneration of 40Gbit/s DPSK signals in a black-box phase sensitive amplifier
}

\author{
Francesca Parmigiani ${ }^{1 *}$, Radan Slavík ${ }^{1}$, Joseph Kakande ${ }^{1}$, Carl Lundström ${ }^{2}$, Martin Sjödin ${ }^{2}$, \\ Peter Andrekson ${ }^{2}$, Ruwan Weerasuriya ${ }^{3}$, Stylianos Sygletos ${ }^{3}$, Andrew D. Ellis ${ }^{3}$, Lars Grüner-Nielsen ${ }^{4}$, \\ Dan Jakobsen ${ }^{4}$, Søren Herstrøm ${ }^{4}$, Richard Phelan ${ }^{5}$, James O'Gorman ${ }^{5}$, Adonis Bogris ${ }^{6}$, Dimitris Syvridis ${ }^{6}$, \\ Sonali Dasgupta ${ }^{1}$, Periklis Petropoulos ${ }^{1}$, and David J. Richardson ${ }^{1}$ \\ 1. Optoelectronics Research Centre, University of Southampton, Southampton, SO17 1BJ, UK \\ 2. Photonics Laboratory, MC2, Chalmers University of Technology, SE-412 96 Göteborg, Sweden \\ 3. Tyndall National Institute and Department of Physics, University College Cork, Cork, Ireland \\ 4. OFS, Priorparken 680, 2605 Brøndby, Denmark \\ 5. Eblana Photonics Inc., Dublin, Ireland \\ 6. University of Athens, Panepistimiopolis, Illisia, Athens, GR-15784, Greece \\ *frp@orc.soton.ac.uk
}

\begin{abstract}
We present a black-box four wave mixing based bit-rate-flexible phase sensitive amplifier and use it in the first demonstration of $40 \mathrm{Gbit} / \mathrm{s}$ DPSK phase regeneration. (C)2010 Optical Society of America

OCIS codes: (060.2320) Fiber optics amplifiers and oscillators, (190.4410) Nonlinear optics, parametric processes
\end{abstract}

\section{Introduction}

Phase noise introduced during transmission both from optical amplifiers and the nonlinear interactions between channels represents a significant limiting factor to data transmission using (differential) phase-shift keying (PSK) modulation formats [1]. Consequently, there is great interest in developing all-optical regeneration schemes capable of eliminating phase (and ideally also amplitude) noise for PSK signals. To date most work on all-optical regeneration has focused on amplitude regeneration of signals and one approach to elimination of phase noise is to adopt some form of phase-to-amplitude format conversion and then to apply amplitude regeneration on the amplitude encoded signal [2]. However, this is a non-optimal solution and results in a complex system - direct elimination of the phase noise would be far more desirable.

Phase regeneration can be implemented directly by exploiting the phase squeezing capability of phase-sensitive amplifiers (PSAs), and amplitude regeneration can be simultaneously performed by operating in the saturated regime. Initial proof of concept results have recently been demonstrated [3, 4]. PSA can be achieved in fiber using either interferometric configurations based on nonlinear optical loop mirrors (NOLMs) [3], or using four-wave mixing (FWM) processes [4] implemented in either single- or dual-pump configurations.

In order to achieve PSA, a phase relationship between the PSA pump(s) and the signal needs to be maintained, which is challenging in practice. In all FWM based PSA regeneration demonstrations reported to date both pump(s) and signal were (artificially) generated from a single (master) continuous wave (CW) laser - typically relying on high frequency electro-optic modulation to generate multiple phase-coherent beams to be used as signal and pumps $[3,4]$. This has the significant drawbacks of limiting the signal regeneration bandwidths to a few tens of GHz and, more crucial, of making the regenerator impractical for use in a real transmission system, where some means to recover the carrier from a carrier-less PSK signal is required and then to phase-lock the necessary pumps to this in order to realize "black-box" operation.

Here, we propose and experimentally demonstrate the first black-box 40Gbit/s phase regenerator. It is based on a dual-pump degenerate PSA configuration in which the two pumps are $\pm 200 \mathrm{GHz}$ apart from the signal carrier frequency. The pumps are generated locally in the regenerator and are phase locked to the recovered signal carrier using a combination of FWM in fiber followed by injection locking of a discrete-mode semiconductor laser [5]. The regenerating PSA is performed in a new type of alumino-silicate strained highly nonlinear fiber (HNLF) whose increased Stimulated Brillouin Scattering (SBS) threshold alleviates the need for any active SBS suppression, significantly improving the system noise performance.

\section{Principle of operation and experimental results}

The setup is shown in Fig. 1. The data signal is a 40Gbit/s non-return-to-zero (NRZ)-DPSK, $2^{31}-1$ pseudo-random bit sequence (PRBS). In order to emulate the effects of phase noise, the signal phase was modulated in a deterministic fashion using an additional phase modulator, driven at a frequency of $\sim 20 \mathrm{GHz}$ and of a controllable modulation depth to emulate different levels of noise. The distorted signal was then launched into the black-box 


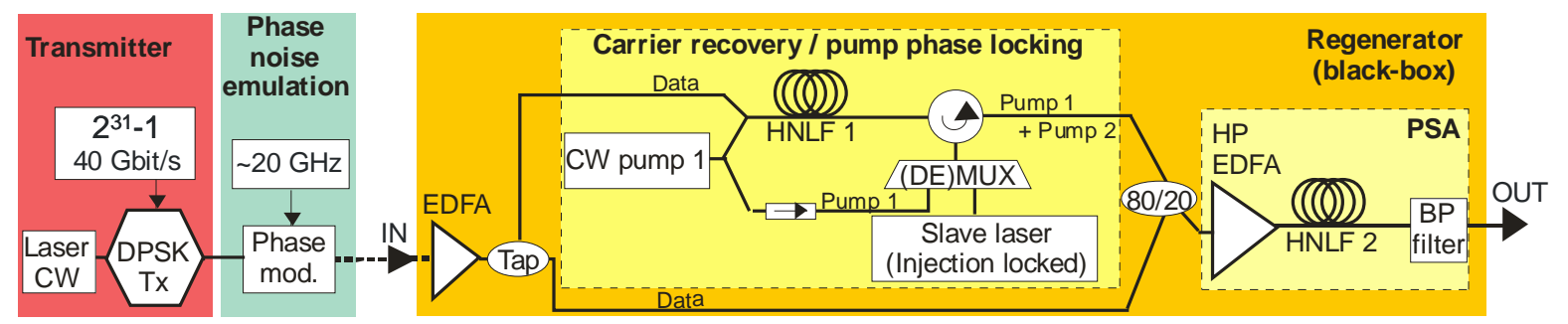

Figure 1: Experimental set-up of the $40 \mathrm{Gbit} / \mathrm{s}$ phase regenerator.

Regenerator Input, Output: No noise
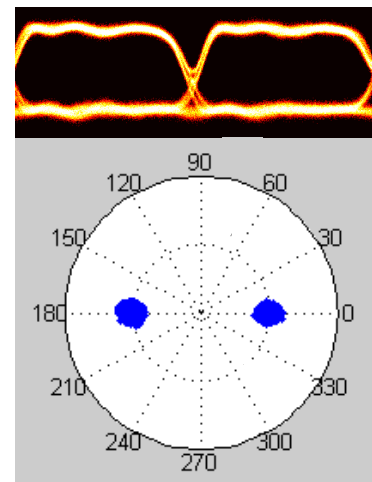
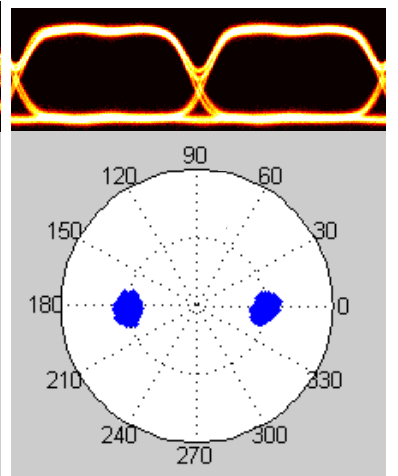

Regenerator Input, Output: High noise level
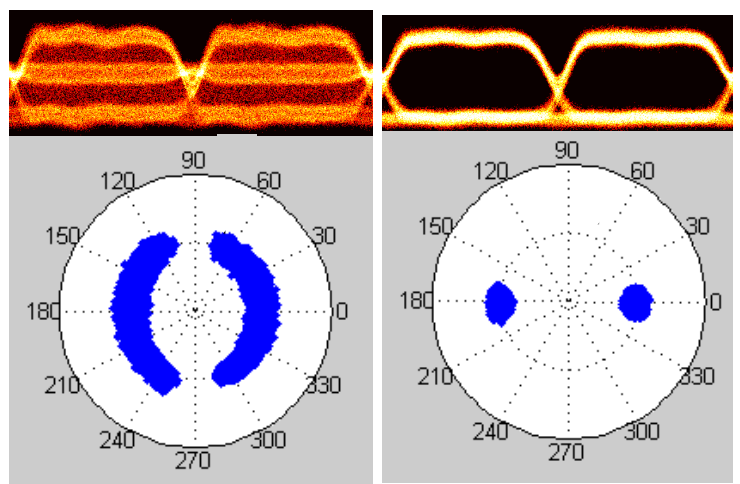

Figure 2: Demodulated eye diagrams after balanced detection and DPSK constellation diagrams (showing amplitude and bit-tobit phase changes) at the input/output of the regenerator measured at $10 \mathrm{Gbit} / \mathrm{s}$.

regenerator. A portion of the signal was initially tapped off to facilitate the frequency and phase locking of the two pumps, which occurs in two steps. First, the tapped signal was mixed in a germano-silicate HNLF with a narrow linewidth CW laser (Pump1) detuned from the signal by $200 \mathrm{GHz}$, to parametrically generate an idler wave that served as the seed for the second pump. The length, dispersion, nonlinear coefficient and attenuation of the HNLF were $500 \mathrm{~m},-0.09 \mathrm{ps} / \mathrm{nm} / \mathrm{km}, 11.5 \mathrm{~W}^{-1} \mathrm{~km}^{-1}$ and $0.83 \mathrm{~dB} / \mathrm{km}$, respectively. Note that due to the phase erasure process, the binary data modulation is not transferred to the idler [5]. Then, the weak idler wave was filtered and injected into a (slave) semiconductor laser (Pump2) by means of a multiplexer [6]. As the injection locking is a much slower process than FWM (having a (sub)-GHz bandwidth), any high frequency fluctuations (e.g., bit-to-bit phase variations) present on the original data signal were not transferred onto the output of the slave laser. At this stage Pump1 and Pump2 were phase locked to the signal and could serve as pumps in a degenerate PSA configuration. They were then coupled together with the data signal and the three signals were amplified to a total power of $\sim 34 \mathrm{dBm}$ and launched into an alumino-silicate strained HNLF for phase regeneration. The length, dispersion, polarization mode dispersion, nonlinear coefficient and attenuation of the fiber are $177 \mathrm{~m},-0.13 \mathrm{ps} / \mathrm{nm} / \mathrm{km}$, $0.11 \mathrm{pskm}^{-0.5}, 7.1 \mathrm{~W}^{-1} \mathrm{~km}^{-1}$ and $15 \mathrm{~dB} / \mathrm{km}$, respectively. The relative powers of the pumps and signal were adjusted for optimal regeneration performance. Any slow (sub $\mathrm{kHz}$ ) relative phase drifts between the interacting waves picked up due to acoustic and thermal effects present prior to the PSA fibre (e.g., in the HNLF that generates the seed of the second pump) were compensated for by an electrical phase-locked loop that controlled a piezoelectric-based fiber stretcher in the pump path (to simplify the schematic, this feedback system is not shown in Fig.1).

The performance of the regenerator was first studied using a constellation analyzer based on a homodyne coherent receiver and offline digital signal processing (DSP) operating at 10Gbit/s [7, 8]. The homodyne approach was chosen over the intradyne one to avoid any artificial phase compression originating from the DSP. The local oscillator used in the measurement was obtained by tapping off the signal laser before data encoding. From these measurements, amplitude and bit-to-bit phase changes were calculated and the corresponding DPSK constellation diagrams are shown in Fig.2. Note that for these measurements, the data rate was adjusted to 10Gbit/s and the phase modulator that distorted the signal was driven at $\sim 5 \mathrm{GHz}[3-4]$. The results show that the phase noise can be squeezed by the regenerator to the back-to-back level and almost negligible amplitude noise is induced even for as extreme peak-to-peak values of phase distortion as $\pm 60 \mathrm{deg}$ (Fig.2). Corresponding bit error ratio (BER) measurements (not shown here) confirmed error free operation with negligible power penalty as compared to the back-to-back even for the most extreme case of added noise reported in Fig.2. 


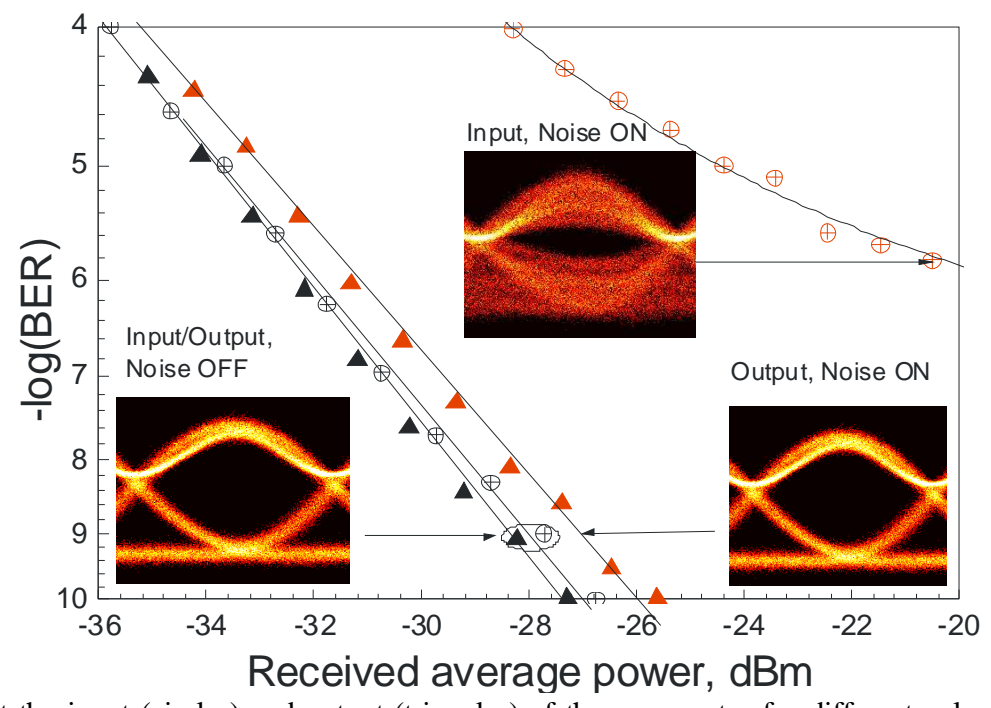

Figure 3: BER curves at the input (circles) and output (triangles) of the regenerator for different values of phase noise added. Inset: Demodulated eye diagrams after balanced detection at the input and output of the regenerator in the absence/presence of phase noise. These measurements were taken at $40 \mathrm{Gbit} / \mathrm{s}$.

The regenerator was then fully assessed at 40Gbit/s using eye diagram and BER measurements for several different levels of added phase noise. In all cases studied, the eye diagrams showed a significant improvement after demodulation at the output of the regenerator and error-free performance was achieved. One such case is presented in Fig.3. Measurement of the phase distorted input signal shows an error floor at the $10^{-6}$ level and a heavily degraded eye diagram. At the output of the regenerator an open eye diagram and error-free performance were achieved with a power penalty of just $\sim 0.5 \mathrm{~dB}$ as compared to the back-to-back case.

\section{Conclusions}

We have proposed and experimentally demonstrated the first black-box 40Gbit/s DPSK phase sensitive amplifier and phase regenerator using degenerate FWM in a high SBS-threshold highly nonlinear fiber. Signal performance approaching the back-to-back one was achieved at the output of the regenerator even for extreme cases of induced phase distortion. The two pumps used in the scheme were phase locked with the incoming signal prior to the PSA using a carrier recovery mechanism employing FWM followed by injection locking. Much higher repetition rates can be envisaged since the proposed configuration allows for far larger values for the pump-signal spacing ( > \pm 200 $\mathrm{GHz}$ ) by simply employing different $\mathrm{CW}$-laser frequencies for the local pump.

This research has received funding from the European Communities Seventh Framework Programme FP/2007-2013 under grant agreement 224547 (PHASORS).

\section{References}

[1] J. P. Gordon, and L. F. Mollenauer, "Phase noise in photonic communications systems using linear amplifiers," Opt. Lett. 15(23), 13511353 (1990).

[2] M. Matsumoto and H. Sakaguchi, "DPSK signal regeneration using a fiber-based amplitude regenerator," Opt. Express 16, 11169-11175 (2008).

[3] K. Croussore, et al., "Demonstration of phase-regeneration of DPSK signals based on phase-sensitive amplification," Opt. Express, 13, 3945-3950 (2005).

[4] K. Croussore and G. Li, “Amplitude regeneration of RZ-DPSK signals based on four-wave mixing in fibre,” Electron. Lett. 43, 177-178 (2007).

[5] G.-W. Lu, et. al., "Optical phase add/drop for format conversion between DQPSK and DPSK and its application in optical label switching systems", IEEE Phot. Technol. Lett. 21, 322-324 (2009).

[6] R. Weerasuriya, et al., "Generation of frequency symmetric signals from a BPSK input for Phase Sensitive Amplification”, OWT6, paper number 1928, OFC 2010.

[7] M. Sjödin, et al., "Cancellation of SPM in Self-Homodyne Coherent Systems," ECOC 2009, paper We8.4.5.

[8] Sköld, et. al., "Constellation diagram analysis of DPSK signal regeneration in a saturated parametric amplifier", Opt. Express 16, 59745982 (2008). 